\title{
Kompressionstherapie bei Patienten mit Ulcus cruris - Welche Kosten entstehen wirklich?
}

\section{Compression Therapy in Patients with Leg Ulcers - which Costs do Really Occur?}

\author{
Autoren \\ Joachim Dissemond ${ }^{1}$, Kerstin Protz ${ }^{2}$, Maurice Moelleken ${ }^{1}$, Knut Kröger ${ }^{3}$
}

Institute

1 Klinik für Dermatologie, Venerologie und Allergologie, Universitätsklinikum Essen

2 Universitätsklinikum Hamburg Eppendorf, Institut für Versorgungsforschung in der Dermatologie und bei Pflegeberufen (IVDP), CWC - Comprehensive Wound Center, Hamburg

3 Klinik für Gefäßmedizin, Angiologie, HELIOS Klinikum Krefeld, Krefeld

Schlüsselwörter

Kompressionstherapie, Ulcus cruris, Entstauungsphase, Erhaltungsphase, Ödeme

Key words

compression therapy, leg ulcer, decongestion phase, conservation phase, edema

Bibliografie

DOI https://doi.org/10.1055/a-1020-2268

Online-Publikation: 7.11.2019

Phlebologie 2020; 49: 79-86

(c) Georg Thieme Verlag KG, Stuttgart · New York ISSN 0939-978X

Zitierweise für diesen Artikel DMW - Deutsche Medizinische Wochenschrift 2019; 144(16): 94-101, DOI:10.1055/a-08419726

Korrespondenzadresse

Prof. Dr. med. Joachim Dissemond

Universitätsklinikum Essen

Klinik und Poliklinik für Dermatologie, Venerologie und

Allergologie, Hufelandstraße 55, 45122 Essen

joachim.dissemond@uk-essen.de

\section{ZUSAMMENFASSUNG}

Hintergrund Patienten mit Ulcus cruris haben häufig ausgeprägte Ödeme der unteren Extremitäten, die im Rahmen einer erfolgreichen Wundversorgung behandelt werden müssen. Die hierfür notwendigen Kompressionstherapien werden heute in Deutschland oft mit sehr fehler- und zeitaufwendigen Verbänden mit Kurzzugbinden durchgeführt. Mehrkomponentensysteme, adaptive Kompressionsbandagen und Ulkus-Strumpfsysteme sind neuere, deutlich weniger fehler- anfällige Therapieoptionen. Neben dem oft nicht vorhandenen Wissen werden auch die scheinbar hohen Kosten als Grund für die nicht vorgenommene Verordnung dieser Materialien angeführt. Es war daher das Ziel unserer Untersuchung, die Kosten der verschiedenen Therapieoptionen im ambulanten und stationären Sektor differenziert darzustellen.

Methoden Für die ökonomischen Berechnungen wurden sowohl die Material- als auch die Personalkosten für verschiedene Szenarien berücksichtigt.

Ergebnisse Es zeigte sich, dass durch die kontinuierliche Kompressionstherapie im stationären Bereich Kosten von 5,29 Euro bis 18,50 Euro pro Tag anfallen. Für die ambulante Versorgung wurden Kosten von 2,29 Euro bis 34,29 Euro pro Tag ermittelt. Die verschiedenen Konstellationen der Kompressionstherapie können somit nicht nur medizinisch, sondern auch wirtschaftlich unterschiedlich sinnvoll sein.

Folgerung Als Konsequenz dieser Daten sollten sowohl die verschiedenen Materialien als auch die ökonomischen Aspekte der Kompressionstherapie bei Patienten mit Ulcus cruris den Therapeuten bekannt sein. Diese Therapieoptionen sollten dann unter Einbeziehung individueller Faktoren und orientiert an den Bedürfnissen und Fähigkeiten der Patienten verordnet und durchgeführt werden.

\section{ABSTRACT}

Introduction Patients with leg ulcers often have severe edema of the lower extremities, which should be treated as part of a successful wound treatment. Today in Germany the necessary compression therapies are often performed with very error-prone and time-consuming short-stretch bandages only. Multicomponent systems, adaptive compression bandages and leg ulcer stocking systems are newer, much less errorprone treatment options. In addition to the often lacking knowledge, the fears of high costs are also mentioned as reasons for the lack of prescription of these systems. It was therefore our aim to investigate the costs of different treatment options, differentiated in the outpatient and inpatient sector. Methods For the economic calculations, both material and personnel costs were taken into account for different scenarios. Results Both material and personnel costs were included in the calculation. We were able to demonstrate that the cost for a continuous compression therapy for inpatients accrues between 5.29 Euros to 18.50 Euros per day. For the outpatient setting 
costs of 2.29 Euros to 34.29 Euros per day were calculated. The different constellations of compression therapy can make sense thus not only for medically but also for economically aspects.

Conclusion As a consequence of this data, both the different systems and the economic factors of compression therapy in patients with leg ulcers should be known to the therapists. These treatment options should then be prescribed and performed according to individual factors, taking into account the needs and abilities of the patients.

\section{Einleitung}

In Deutschland leiden etwa eine Million Menschen unter einem chronischen Ulcus cruris [1]. Hierfür können viele verschiedene Ursachen, Komorbiditäten und Kofaktoren verantwortlich sein. Bei etwa 80 \% dieser Patienten liegen Erkrankungen der Venen und/oder Arterien zugrunde [2]. Patienten mit chronischem Ulcus cruris haben oft auch ausgeprägte Ödeme der unteren Extremitäten. Jede Art von Ödem kann an der betroffenen Extremität zu einer erheblichen Störung der Wundheilung führen. Somit ist die Beseitigung dieser Ödeme eine Grundvoraussetzung für die adäquate Heilung. Für Ödeme, die durch eine chronische venöse Insuffizienz (CVI) und/oder ein Lymphödem bedingt sind, ist dies wissenschaftlich gut belegt. Die Notwendigkeit der Behandlung von Ödemen gilt aber ebenso für alle anderen Ursachen. Hier kommt der Kompressionstherapie als physikalische, nebenwirkungsarme Therapieoption die größte Bedeutung zu [3]. Es stehen für die Behandlung der Patienten mit Ulcus cruris heute viele verschiedene Materialien für die Durchführung einer Kompressionstherapie zur Verfügung, die unterschiedliche Vor- und Nachteile haben [4].

Gerade im deutschsprachigen Raum hat die Kompressionstherapie eine sehr lange Tradition [5]. Dennoch belegen aktuelle Untersuchungen eine eklatante Unterversorgung. So zeigte die Analyse der Datensätze einer großen deutschen Krankenkasse, dass lediglich 40,6\% der Patienten mit floridem Ulcus cruris eine Kompressionstherapie verordnet bekommen haben. Hiervon erhielten lediglich 3,1\% ein Mehrkomponentensystem [6]. Neben dem oft nicht vorhandenen Wissen werden vielfach auch die scheinbar hohen Kosten als Grund für die nicht vorgenommene Verordnung, insbesondere von „neuen“ Kompressionsmaterialien, angeführt.

Es war daher das Ziel unserer Untersuchung, die tatsächlich auftretenden Kosten für die verschiedenen Therapieoptionen der Kompressionstherapie im ambulanten und stationären Sektor differenziert darzustellen.

\section{Materialien und Methoden}

\section{Berechnungsgrundlage}

Der Schwerpunkt dieser Untersuchung lag auf der Ermittlung der durch Kompressionstherapie direkt verursachten Kosten für Materialien und Personal. Für die Beantwortung dieser Fragestellung mussten im Vorfeld verschiedene Sachverhalte geklärt werden. Um eine vergleichbare Berechnungsgrundlage zu schaffen, wurden daher von den Autoren verschiedene Fakten auf Basis aktueller wissenschaftlicher Erkenntnisse und praktischer Erfahrungen diskutiert und zugrunde gelegt [5, 7-9]:
- Für die initiale Entstauungsphase können Kompressionsverbände mit Kurzzugbinden, Mehrkomponentensystemen oder adaptiven Kompressionsbandagen eingesetzt werden.

- Für die Entstauungsphase wurde ambulant ein Zeitraum von vier Wochen angesetzt.

- In der anschließenden Erhaltungsphase sollte, bis zu der vollständigen Abheilung des Ulcus cruris, die Behandlung mit adaptiven Kompressionsbandagen fortgeführt werden oder alternativ ein Ulkus-Strumpfsystem zum Einsatz kommen.

- Kurzzugbinden werden stationär lediglich einmal verwendet und dann verworfen, da die Aufarbeitung im Krankenhaus teurer als die Neubeschaffung ist.

- Ambulant werden Kurzzugbinden meist mehrfach verwendet. Bei adäquater Pflege können die Binden etwa 15- bis 20-mal gewaschen werden, bis sie ausgeleiert sind und ausgetauscht werden sollten. Ein vorkonfektioniertes Kompressions-Set kann somit für die komplette Entstauungsphase von vier Wochen genutzt werden. Solche Sets enthalten meist vier Schaumstoffpolsterbinden, eine Rolle Schlauchverband, ein Rollenpflaster, ein Wäschenetz sowie jeweils zwei Kurzzugbinden in $8 \mathrm{~cm}$ und in $10 \mathrm{~cm}$ Breite.

- Verbände mit Kurzzugbinden sollten grundsätzlich unterpolstert werden. Hierfür werden stationär meist Polsterwatte als Einmalmaterial und ambulant oft Schaumstoffpolsterbinden für die mehrfache Anwendung genutzt. Letztere sind ebenfalls nach jedem Verbandwechsel zu waschen.

- Für einen Verband mit Kurzzugbinden wurden jeweils zwei Binden in $10 \mathrm{~cm}$ Breite à $3 \mathrm{~m}$ Polsterwatte beziehungsweise à 2,5 m Schaumstoffpolsterbinde pro Verband berechnet.

- Bei Verbänden mit Kurzzugbinden sind Schlauchverbände als Einmalmaterial für den Schutz der Haut zu verwenden.

- Für einen Verband mit Kurzzugbinden wurde eine Länge von 1,7 m Schlauchverband berechnet.

- Mehrkomponentensysteme können nur einmal angewendet werden.

- Mehrkomponentensysteme gewährleisten den therapierelevanten Kompressionsdruck, je nach Entstauungssituation, bis zu einer Woche. Bei Patienten mit Ulcus cruris sind jedoch insbesondere das Exsudataufkommen und der daher resultierende Verbandwechsel zeitlich limitierend.

- Adaptive Kompressionsbandagen können, ebenso wie UlkusStrumpfsysteme, bei adäquater Pflege bis zu sechs Monate verwendet werden.

- Für die Verbände mit Kurzzugbinden muss im ambulanten Bereich aufgrund hygienischer Aspekte die Möglichkeit der Wechselversorgung gegeben sein. 
- Bei adaptiven Kompressionsbandagen werden ebenso wie bei Ulkus-Strumpfsystemen für die Wechselversorgung zwei Unterstrümpfe mitgeliefert.

- Kompressionsversorgungen mit Binden sollten durch gut geschulte Pflegefachkräfte und keinesfalls durch Patienten selbst angelegt werden.

- Für das An- und Ablegen von Ulkus-Strumpfsystemen benötigen viele Patienten im ambulanten Bereich einen Pflegedienst oder zumindest An- und Ausziehhilfen.

- Adaptive Kompressionsbandagen können bei entsprechender körperlicher Voraussetzung meist von Patienten oder Angehörigen nach kurzer Schulung selbstständig an- und ausgezogen sowie im Tagesverlauf nachjustiert werden.

- Ein Ulcus cruris sollte nach mehreren Wochen Behandlung vollständig abgeheilt sein. In der täglichen Praxis gelingt dies nicht immer und einige Wunden persistieren über mehrere Jahre. Für eine bessere Vergleichbarkeit wurde jeweils ein Behandlungszeitraum von sechs Monaten (180 Tage) berechnet.

- Für die stationäre Behandlung wurde ein Zeitraum von jeweils 7 Tagen berechnet.

\section{Materialkosten}

Für die Kalkulation der Materialkosten wurden die Verkaufspreise aus der Lauer-Taxe exemplarisch für weit verbreitete Kompressionsprodukte verwendet. Diese Preise können für andere Systeme oder im Rahmen von Rabattverträgen abweichen. Die Preise im stationären Bereich wurden auf der Grundlage persönlicher Kommunikation entsprechend der in verschiedenen Krankenhäusern tatsächlich anfallenden Kosten ermittelt und variieren ebenso ( $\vee$ Tab. 1).

\section{Personalkosten}

Für den stationären Bereich gibt es keine separate Erstattung, beispielsweise über die Ziffern des Operationen- und ProzedurenSchlüssel (OPS), für die Kompressionstherapie. Personalkosten entstehen hier in erster Linie durch den Zeitaufwand der Pflege. In der Hautklinik am Universitätsklinikum Essen hat ein wissenschaftlicher Mitarbeiter Pflegefachkräfte auf den Stationen im Frühdienst begleitet und bei insgesamt 30 Verbandwechseln die Zeiten gemessen, die für den Gesamtprozess der Anlage von Kompressionsverbänden anfielen. Die ermittelte durchschnittliche Gesamtzeit betrug für Mehrkomponentenverbände 5:18,6 min (SD 0:55,6; Vorbereitung 1:00,9 min; Anlegen 3:57,8 min; Aufräumen 0:20,0 min) und für Verbände mit Kurzzugbinden 12:37,1 min (SD 3:23,2; Vorbereitung 1:51,6 min; Anlegen 9:38,0 min; Aufräumen 1:07,5 min). Für die Berechnung der Personalkosten in der Klinik wurden die Arbeitgeberbruttokosten im Durchschnitt für Mitarbeiter der Pflege am Universitätsklinikum Essen ermittelt. Diese Kosten entsprechen somit nicht dem Bruttogehalt des Mitarbeiters, sondern den tatsächlich entstehenden Kosten für den Arbeitgeber. Hier sind somit u. a. die Sozialabgaben miterfasst. Die bei der Personalabteilung abgefragten Daten ergeben voraussichtliche Kosten für 2017 von 56726,02 Euro/Jahr/Mitarbeiter. Somit wurden Kosten für die Mitarbeiter der Pflege von 28,24 Euro/Stunde beziehungsweise 0,47 Euro/Minute ermittelt.

Im ambulanten Bereich kann es für die Anlage von Kompressionsverbänden oder Strumpfsystemen erforderlich sein, dass die
- Tab. 1 Materialkosten für Kompressionstherapie.

\begin{tabular}{|c|c|c|}
\hline & ambulant & stationär \\
\hline Polsterwatte $^{1}$ & entfällt & $0,30 €$ \\
\hline konfektioniertes Kompressionsset ${ }^{2}$ & $97,22 €$ & entfällt \\
\hline Schlauchverband ${ }^{3}$ & entfällt & $2,70 €$ \\
\hline Kurzzugbinde ${ }^{4}$ & entfällt & $2,-€$ \\
\hline Mehrkomponentensystem ${ }^{5}$ & $29,75 €$ & $8,-€$ \\
\hline adaptive Kompressionsbandage ${ }^{6}$ & $205,67 €$ & entfällt \\
\hline Ulkus-Strumpfsystem ${ }^{7}$ & $68,56 €$ & entfällt \\
\hline An- und Ausziehhilfe ${ }^{8}$ & $52,-€$ & entfällt \\
\hline \multicolumn{3}{|c|}{$\begin{array}{l}1 \text { Wattebinde Cellona, } 10 \mathrm{~cm} \times 3 \text { m, Fa. Lohmann \& Rauscher. } \\
2 \text { Comprilan Plus (4 Kurzzugbinden, Schlauchverband, } 2 \text { Schaumstoff- } \\
\text { binden, Pflaster, Wäschenetz), Fa. BSN Medical. } \\
{ }^{3} \text { tg Schlauchverband, Größe 7, } 20 \text { m, Fa. Lohmann \& Rauscher. } \\
4 \text { Pütter Binde, } 10 \mathrm{~cm} \times 5 \text { m, Fa. Paul Hartmann. } \\
{ }^{5} \text { UrgoK2, } 10 \text { cm, Fa. Urgo. } \\
{ }^{6} \text { Circaid JuxtaCures, Standard, Fa. Medi. } \\
{ }^{7} \text { Venotrain Ulcer Tec, Fa. Bauerfeind (Anmerkung: EK 57,13, VK+ } 20 \% \text { ). } \\
{ }^{8} \text { Magnide on/off, Fa. Sigvaris/Arion. }\end{array}$} \\
\hline
\end{tabular}

oft älteren und multimorbiden Patienten Hilfe durch einen Pflegedienst erhalten. Verbände mit Kompressionsbinden sollten grundsätzlich nicht von den Patienten selbst angelegt werden. Daher wurden bei Verbänden mit Kompressionsbinden für jede Neuanlage die Kosten für einen Pflegedienst berücksichtigt. Die Abrechnung der ambulanten Pflege ist bundesweit sowie auch innerhalb der Bundesländer nicht einheitlich geregelt. Für unsere Berechnungen haben wir die aktuellen Daten der Dienste der freien Wohlfahrtspflege sowie der privat-gewerblichen Pflegedienste in Bayern für die Abrechnung der Versorgung von Patienten, die bei Ersatzkassen (vdek) sowie AOK, IKK, Knappschaft versichert sind, zugrunde gelegt:

- Anziehen von medizinischen Kompressionsstrümpfen: 4,80€ für beide Beine

- Ausziehen von medizinischen Kompressionsstrümpfen: 4,80€ für beide Beine

- Anlegen eines phlebologischen Kompressionsverbandes an einem Bein: 4,80€ pro Bein

- Abnehmen eines phlebologischen Kompressionsverbandes an einem Bein: 2,40€ pro Bein

- Hausbesuchsgebühr: 5,22€ pro Hausbesuch von 8 bis 22 Uhr

Somit entstehen für das An- und Ausziehen von medizinischen Kompressionsstrümpfen (MKS) an zwei Beinen inklusive der Wegepauschale Kosten von 14,82 Euro/Tag. Für phlebologische Kompressionsverbände mit Kompressionsbinden an zwei Beinen sind es entsprechend 24,84 Euro, wenn an einem Tag tatsächlich die Verbände angelegt und abends auch wieder abgenommen werden. Wenn die Kompressionsversorgung zumindest über 24 Stunden belassen wird, sind es an zwei Beinen Kosten für das Abnehmen und die erneute Anlage phlebologischer Kompressionsverbände von 19,62 Euro/Tag. 
- Tab. 2 Zeitvolumen, das von Pflegefachkräften benötigt wurde, um bei stationären Patienten mit Ulcus cruris jeweils beide Unterschenkel mit Kompressionsverbänden zu versorgen. Alle Zeiten sind in Minuten angegeben.

\begin{tabular}{|c|c|c|c|c|c|c|c|c|}
\hline \multirow[b]{2}{*}{ Messung } & \multicolumn{4}{|c|}{ Mehrkomponentenverband } & \multicolumn{4}{|c|}{ Verband mit Kurzzugbinden } \\
\hline & Vorbereitung & Anlegen & Aufräumen & Gesamtzeit & Vorbereitung & Anlegen & Aufräumen & Gesamtzeit \\
\hline 1 & $02: 34,3$ & $04: 41,6$ & $00: 16,5$ & $07: 32,3$ & $01: 20,5$ & $10: 04,7$ & 02:01,7 & $13: 26,9$ \\
\hline 2 & $01: 43,6$ & 03:49,0 & $00: 17,0$ & 05:49,6 & $02: 40,9$ & $12: 24,0$ & $00: 52,0$ & $15: 56,9$ \\
\hline 3 & $00: 40,9$ & $04: 25,1$ & $00: 14,6$ & $05: 20,6$ & $02: 35,2$ & $06: 49,1$ & $00: 43,8$ & $10: 08,2$ \\
\hline 4 & $00: 46,5$ & $03: 52,7$ & $00: 10,4$ & $04: 49,5$ & 01:03,9 & $08: 55,6$ & $01: 12,5$ & $11: 12,0$ \\
\hline 5 & $01: 36,1$ & $05: 23,4$ & $00: 12,9$ & $07: 12,5$ & $01: 44,2$ & 09:43,9 & $00: 46,8$ & $12: 14,9$ \\
\hline 6 & $00: 54,4$ & 04:01,5 & $00: 07,5$ & 05:03,3 & $02: 04,8$ & $08: 38,0$ & $00: 41,4$ & $11: 24,2$ \\
\hline 7 & $00: 55,4$ & $03: 58,4$ & $00: 29,6$ & $05: 23,4$ & $01: 55,1$ & $10: 21,7$ & $00: 51,3$ & $13: 08,1$ \\
\hline 8 & $00: 48,4$ & $03: 40,5$ & $00: 29,0$ & $04: 57,9$ & $02: 13,8$ & $11: 01,9$ & $01: 15,2$ & $14: 30,9$ \\
\hline 9 & $01: 14,2$ & $04: 41,3$ & $00: 22,7$ & $06: 18,3$ & $01: 45,3$ & $09: 50,7$ & $01: 51,0$ & $13: 27,0$ \\
\hline 10 & $00: 34,5$ & $03: 12,3$ & $00: 17,1$ & 04:03,9 & $01: 12,7$ & $08: 30,2$ & $00: 59,1$ & $10: 42,0$ \\
\hline 11 & 01:03,6 & $03: 28,3$ & $00: 26,9$ & $04: 58,7$ & & & & \\
\hline 12 & $01: 03,4$ & $03: 38,5$ & $00: 26,0$ & 05:07,9 & & & & \\
\hline 13 & $00: 48,5$ & 04:07,4 & $00: 21,5$ & $05: 17,3$ & & & & \\
\hline 14 & $00: 41,2$ & 03:30,1 & $00: 18,9$ & $04: 30,2$ & & & & \\
\hline 15 & $00: 37,9$ & $05: 10,4$ & $00: 19,2$ & $06: 07,5$ & & & & \\
\hline 16 & $00: 50,8$ & $03: 12,4$ & $00: 12,9$ & $04: 16,1$ & & & & \\
\hline 17 & $00: 32,5$ & $03: 44,8$ & $00: 35,2$ & $04: 52,5$ & & & & \\
\hline 18 & 01:09,3 & $04: 11,2$ & $00: 37,7$ & $05: 58,2$ & & & & \\
\hline 19 & $00: 55,7$ & $03: 21,3$ & $00: 10,6$ & $04: 27,6$ & & & & \\
\hline 20 & $00: 46,5$ & 03:05,9 & $00: 12,8$ & 04:05,2 & & & & \\
\hline Durchschnitt & 01:00,9 & $03: 57,8$ & $00: 20,0$ & 05:18,6 & $01: 51,6$ & 09:38,0 & 01:07,5 & $12: 37,1$ \\
\hline
\end{tabular}

\section{Ergebnisse}

\section{Zeitvolumen}

Es wurde die benötigte Zeit für die Vor- und Nachbearbeitung sowie das Anlegen von phlebologischen Kompressionsverbänden an jeweils zwei Unterschenkeln ermittelt. Hierfür wurden sieben verschiedene erfahrene Pflegefachkräfte auf einer dermatologischen Station im Universitätsklinikum Essen begleitet. Es konnten die Zeiten für insgesamt 20 Patienten, die Kompressionsbandagierungen mit Mehrkomponentensystemen erhielten, und von 10 Patienten, die Kompressionsbandagierungen mit Kurzzugbinden erhielten, ermittelt werden ( $>$ Tab. 2). Die Pflegefachkräfte haben durchschnittlich 12 Minuten und 37 Sekunden für die Anlage von zwei phlebologischen Kompressionsverbänden mit Kurzzugbinden inklusive Vor- und Nachbereitung aufgewendet ( Abb. 1), woraus Personalkosten von 5,93 Euro resultieren. Für die Mehrkomponentensysteme wurden hingegen lediglich fünf Minuten und 19 Sekunden aufgewendet, sodass hier Personalkosten von 2,50 Euro anfallen.

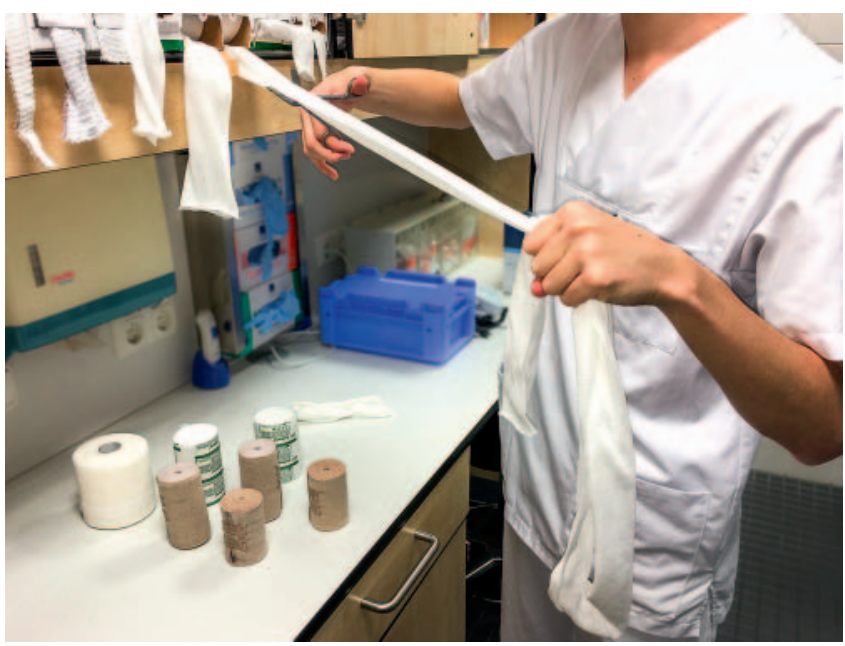

Abb. 1 Vorbereitung eines Kompressionsverbandes mit Kurzzugbinden. 


\section{Berechnung von Kosten in verschiedenen Szenarien}

Patienten sind durch ihre soziale Situation, individuelle Faktoren sowie Fähigkeiten sehr unterschiedlich geprägt. Es wurden daher verschiedene Szenarien kalkuliert, die eine realistische Einschätzung bei einem Großteil der versorgten Patienten ermöglichen sollen. Die Berechnung erfolgte für die Versorgung von zwei Unterschenkeln. Ambulant wurde auch eine unbedingt notwendige Wechselversorgung berechnet.

Kompressionstherapie beider Unterschenkel bei ambulanten Patienten über sechs Monate.

Phlebologische Kompressionsverbände mit Kurzzugbinden

Kompressionsverbände werden morgens von einem Pflegedienst angelegt und abends abgenommen.

$5637,74 €=31,32 € /$ Tag

12-mal Kompressionsset (1166,54€); 180-mal Pflegedienst Binden [2-mal tgl.] (4471,20€).

Phlebologische Kompressionsverbände werden für vier Wochen jeweils 24 Stunden belassen, dann Umstellung auf Ulkus-Strumpfsystem (täglicher Wechsel durch Pflegedienst).

$3133,56 €=17,41 € /$ Tag

2-mal Kompressionsset (194,44€); 2-mal Ulkus-Strumpfsystem (137,12€); 28-mal Pflegedienst Binden (549,36€), 152-mal Pflegedienst Strümpfe $(2252,64 €)$.

Phlebologische Kompressionsverbände werden für vier Wochen jeweils 24 Stunden belassen, dann Umstellung auf Ulkus-Strumpfsystem (Wechsel selbstständig mit An- und Ausziehhilfe).

$932,92 €=5,18 € /$ Tag

2-mal Kompressionsset (194,44€); 2-mal Ulkus-Strumpfsystem (137,12€); 1-mal An- und Ausziehhilfe (52,00€); 28-mal Pflegedienst Binden (549,36€).

Phlebologische Kompressionsverbände mit Mehrkomponentensystemen Wechsel 3-mal/Woche, Pflegedienst notwendig.

$6171,36 €=34,29 € /$ Tag

156-mal Mehrkomponentensystem (4641,00€); 78-mal Pflegedienst Binden (1530,36€).

Wechsel 2-mal Woche, Pflegedienst notwendig.

$4114,24 €=22,86 € /$ Tag

104-mal Mehrkomponentensystem (3094,00€); 52-mal Pflegedienst Binden $(1020,24 €)$.

Behandlung 3-mal/Woche mit Pflegedienst für vier Wochen, dann Umstellung auf Ulkus-Strumpfsystem (täglicher Wechsel durch Pflegedienst).

$3339,20 €=18,55 € /$ Tag

24-mal Mehrkomponentensystem (714,00€); 2-mal UlkusStrumpfsystem (137,12€); 12-mal Pflegedienst Binden (235,44€), 152-mal Pflegedienst Strümpfe (2252,64€).
Behandlung 2-mal/Woche mit Pflegedienst für vier Wochen, dann Umstellung auf Ulkus-Strumpfsystem (täglicher Wechsel durch Pflegedienst).

$3201,04 €=17,78 € /$ Tag

16-mal Mehrkomponentensystem (476,00€); 2-mal UlkusStrumpfsystem (137,12€); 8-mal Pflegedienst Binden (189,36€), 152-mal Pflegedienst Strümpfe $(2398,56 €)$.

Behandlung 3-mal/Woche mit Pflegedienst für vier Wochen, dann Umstellung auf Ulkus-Strumpfsystem (Wechsel selbstständig mit An- und Ausziehhilfe).

$1138,56 €=6,33 € / \mathrm{Tag}$

24-mal Mehrkomponentensystem (714,00€); 2-mal UlkusStrumpfsystem (137,12€); 1-mal An- und Ausziehhilfe $(52,00 €)$; 12-mal Pflegedienst Binden (235,44€).

Phlebologische Kompressionsverbände mit adaptiven Kompressionsbandagen

Wechsel alle 24 Stunden, Selbstmanagement möglich.

$411,34 €=2,29 € /$ Tag

2-mal adaptive Kompressionsbandagen $(411,43 €)$.

Phlebologische Kompressionstherapie beider Unterschenkel bei stationären Patienten über 7 Tage.

Kompressionsverband mit Kurzzugbinden

Wechsel alle 24 Stunden

$109,36 €=15,62 € / \mathrm{Tag}$

28-mal Kurzzugbinde $+25,2$ m Schlauchverband +28 Wattebin-

den (Material: 67,85€) + 7-mal Pflege $(41,51 €)$.

Phlebologischer Kompressionsverband mit Mehrkomponentensystem Wechsel alle 24 Stunden

$129,50 €=18,50 € /$ Tag

14-mal Mehrkomponentensystem $(112,00 €)+7$-mal Pflege $(17,50 €)$.

Wechsel 3-mal/Woche

$55,50 €=7,93 € /$ Tag

6-mal Mehrkomponentensystem $(64,00 €)+3$-mal Pflege $(7,50 €)$.

Wechsel 2-mal/Woche

$37,00 €=5,29 € /$ Tag

4-mal Mehrkomponentensystem $(32,00 €)+2$-mal Pflege $(5,00 €)$.

\section{Diskussion}

Die adäquat durchgeführte Kompressionstherapie ist eine nebenwirkungsarme, wissenschaftlich gut belegte Säule der erfolgreichen Behandlung von Patienten mit Ulcus cruris und Ödemen [5]. Hierfür stehen heute verschiedene Materialien zur Verfügung.

\section{Materialien für die Kompressionstherapie bei Ulcus cruris}

Grundsätzlich ist in der Behandlung von Ödemen die initiale Entstauungsphase von der darauffolgenden Erhaltungsphase zu unterscheiden. Für die Entstauungsphase sind prinzipiell die verschie- 
denen Systeme mit Kompressionsbinden sowie die adaptiven Kompressionsbandagen geeignet. In der Erhaltungsphase ist dann auf eine Versorgung mit Ulkus-Strumpfsystemen umzustellen. Alternativ kann die Behandlung mit adaptiven Kompressionsbandagen fortgeführt werden [3, 4].

Im deutschsprachigen Raum werden bei Patienten mit Ulcus cruris für die Ödemtherapie meist Kompressionsverbände mit Kurzzugbinden ausgeführt, die mit einem Ruhedruck von 40 bis $60 \mathrm{mmHg}$ angelegt werden sollten. Diese Verbände sind sehr fehleranfällig und erfordern für die korrekte Anlage eine gute Schulung und praktische Erfahrung aufseiten der Versorger.

Bei den Mehrkomponentensystemen handelt es sich um vorgefertigte Bindensysteme, die aus Polster-, Kompressions- und Fixierbinden bestehen. Für die Anlage dieser Systeme sind keine besonderen Kompressionstechniken erforderlich. Dadurch sind sie weniger fehleranfällig. Einige dieser Systeme verfügen über spezielle Dehnungstechniken oder haben visuelle Markierungen auf den Binden, die eine Orientierung geben sollen, wann die korrekte Vordehnung und somit der richtige Anlagedruck erreicht werden. Als äußere Lage wird meist eine kohäsive Binde verwendet, die sowohl die Unnachgiebigkeit verstärkt als auch den Kompressionsdruck der Verbände bis zu einer Woche, je nach Entstauungssituation, gewährleistet.

Die auf dem deutschen Markt neuen adaptiven Kompressionsbandagen werden über mehrere Klettverschluss-Bänder an den Unterschenkeln angelegt und fixiert. Bei einigen Systemen kann über Markierungen und eine Schablone der Druck definiert werden. Die Füße werden bei diesem System separat mit Kompressionssocken versorgt.

Ulkus-Strumpfsysteme bestehen aus einem Unterziehstrumpf mit einem geringen Anpressdruck, der den Wundverband fixiert und bei einigen Systemen gleichzeitig als Anziehhilfe für den zweiten Strumpf dient. Darüber wird dann der eigentliche medizinische Kompressionsstrumpf angezogen. Beide Strümpfe zusammen erreichen bei den meisten dieser Systeme mindestens $40 \mathrm{mmHg}$, was einer Kompressionsklasse III entspricht [5].

\section{Behandlungsqualität}

Obwohl die adäquate Ödemtherapie eine Grundvoraussetzung für die Wundheilung ist, erhält aktuell in Deutschland weniger als die Hälfte der Patienten mit floridem Ulcus cruris eine Kompressionstherapie [6]. Aber selbst wenn eine Kompressionstherapie durchgeführt wird, ist die Qualität nicht immer sichergestellt $[10,11]$. So zeigte eine aktuelle Analyse der Druckwerte von Kompressionsverbänden mit Kurzzugbinden, die durch 972 zertifizierte Wundexperten im Rahmen von Fortbildungen in Deutschland angelegt wurden, Resultate zwischen 6 und $173 \mathrm{mmHg}$. Nur $12,2 \%$ der Probanden erzielten den vorgegebenen optimalen Kompressionsdruck von 50 bis $60 \mathrm{mmHg}$ [6]. Als Konsequenz aus diesen Daten ergibt sich, dass bei der Anlage von Kompressionsverbänden die erzielten Druckwerte, abhängig von der Erfahrung und den praktischen Fähigkeiten, möglichst mit Druckmesssonden überprüft werden sollten. Bei den Mehrkomponentensystemen gibt es Materialien mit speziellen Dehnungstechniken oder aufgedruckten Markierungen, die den Anwendern eine Orientierungshilfe für die adäquate Vordehnung und Anlage bieten kön-

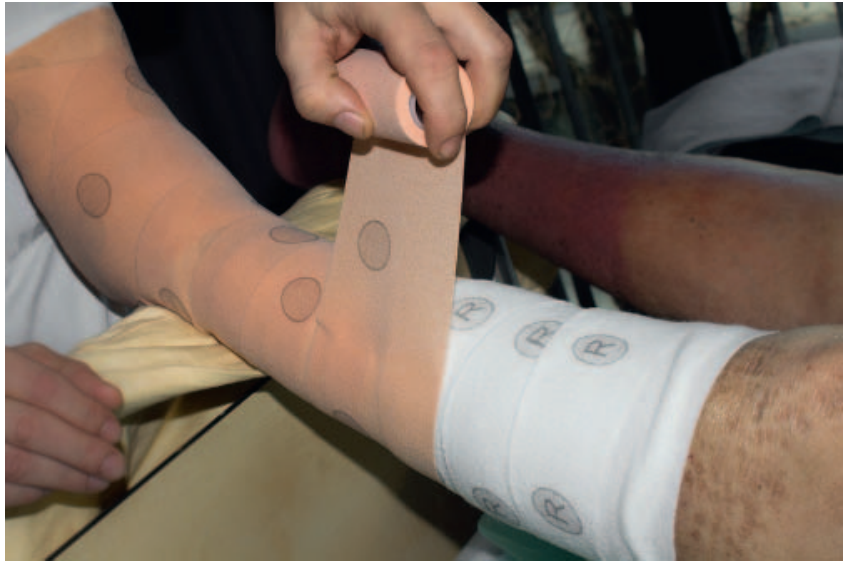

Abb. 2 Anlegen eines Mehrkomponentensystems mit Markierungen für die Kompressionstherapie.

nen ( $\triangleright$ Abb. 2). In einer prospektiven Studie konnte gezeigt werden, dass nicht nur Ärzte und Pflegefachkräfte, sondern auch Laien mit diesen Systemen, im Gegensatz zu den Verbänden mit Kurzzugbinden, meist adäquate Kompressionsverbände anlegen können [12]. Eine aktuelle Studie mit 31 Probanden belegt, dass alle Patienten nach kurzer Schulung in der Lage waren, einen optimalen Kompressionsdruck mit der Anwendung von adaptiven Kompressionsbandagen zu erzielen. Dieser Druck war auch nach zwei Tagen weiterhin im Optimalbereich [13]. Ulkus-Strumpfsysteme gewährleisten bei adäquater Vermessung und Pflege eine optimale Kompressionstherapie [14]. In einer aktuellen Studie wurde der Tragekomfort verschiedener Kompressionssysteme verglichen. Als „angenehm“ wurde der Tragekomfort bei Kompressionsverbänden mit Kurzzugbinden von 37,7\%, bei Mehrkomponentensystem von $65 \%$ und bei adaptiven Kompressionsbandagen von 94,6\% der 137 Teilnehmer beschrieben. Dieser Aspekt ist insbesondere im Hinblick auf die Akzeptanz und Adhärenz der Patienten wichtig. Diese Studie bestätigt zudem die bereits erwähnten Aussagen zu der Anlagequalität der einzelnen Therapieoptionen. Lediglich 11,2\% der 134 Kompressionsversorgungen mit Kurzzugbinden lagen innerhalb der vorgegebenen Zieldruckwertspanne. Bei Mehrkomponentensystemen waren dies 35,2\% und bei der adaptiven Kompressionsbandage 85 \% [15].

\section{Kompressionstherapie bei Ulcus cruris - Was ist sinnvoll?}

Es gibt keine Kompressionstherapie, die für alle Patienten mit Ulcus cruris und Ödemen optimal geeignet erscheint. Bei der Auswahl der geeigneten Materialien sind individuelle Faktoren und Komorbiditäten ebenso wie die Präferenzen und Fähigkeiten der Patienten zu berücksichtigen [16]. Zusätzlich können und sollten zukünftig auch ökonomische Aspekte beachtet werden. Ein eindrückliches Beispiel hierfür ist die in Deutschland weiterhin sehr weit verbreitete Praxis, bei den Patienten morgens durch einen Pflegedienst Kompressionsverbände mit Kurzzugbinden anzulegen und abends wieder zu entfernen. Dieses Vorgehen ist weder medizinisch noch ökonomisch betrachtet sinnvoll. Läuft der Patient abends nach Ablegen der Kompressionsversorgung noch umher oder ist morgens bereits vor Anlegen in Bewegung, geht 
der erzielte Entstauungserfolg des Vortags wieder verloren. In der Folge werden viele Patienten so über Monate, teils über Jahre ohne ausreichenden Erfolg behandelt [17]. Dies bestätigt auch eine aktuelle Studie, in der Patienten mit Ulcus cruris venosum im Durchschnitt über 40 Wochen mit phlebologischen Kompressionsverbänden mit Kurzzugbinden versorgt wurden [7].

Wenn Wunden in der Entstauungsphase sehr exsudativ sind, dann sollte zumindest bei den Patienten, bei denen die Exsudataufnahme der Wundauflagen erschöpft ist, ein Wechsel der Verbände nach 24 Stunden erfolgen. Auf der einen Seite erscheinen insbesondere bei ambulanten Patienten Mehrkomponentensysteme aufgrund der höheren Kosten nicht die geeigneten Materialien zu sein, auf der anderen Seite lässt durch eine rasche suffiziente Kompression die Exsudation oft schnell nach, sodass in Einzelfällen auch hier eine Kompressionstherapie mit Mehrkomponentensystemen über mehrere Tage versucht werden kann. Im Laufe der Behandlung sollte unter Berücksichtigung der ökonomischen Aspekte dann aber ein Wechsel lediglich 3-mal, besser 2-mal pro Woche erfolgen. Mehrkomponentensysteme sind dann auch im ambulanten Sektor in der Entstauungsphase eine Therapieoption der ersten Wahl. Im stationären Bereich stellt sich die Situation anders dar. Hier sind die Materialkosten erheblich geringer. Insbesondere bei Mehrkomponentensystemen erhöht sich die Behandlungssicherheit im Vergleich zu der Anlage von Kompressionsverbänden mit Kurzzugbinden. Ein weiterer Vorteil ist die Option der Verwendung von sogenannten „lite“-Systemen, bei denen die Kompressionstherapie mit geringeren Druckwerten um $20 \mathrm{mmHg}$ durchgeführt werden kann [4, 18]. Die von uns ermittelten Zeiten für das Anlegen von Kompressionsverbänden sind in etwa mit Daten aus anderen Untersuchungen vergleichbar [15]. Es zeigt sich, dass durch die Verwendung von Mehrkomponentensystemen im Vergleich zu Verbänden mit Kurzzugbinden durchschnittlich sieben Minuten und 18 Sekunden eingespart werden können ( Tab.2). Diese Differenz entspricht in Bezug auf die Personalkosten im stationären Bereich einer Einsparung von 3,43 Euro/Verband. Im ambulanten Bereich hilft diese Zeitersparnis insbesondere den Pflegediensten, da die Vergütung der verschiedenen Verbandsysteme identisch ist.

Bei Ulkus-Strumpfsystemen sollte mit den Patienten im Vorfeld geklärt werden, ob eine Anlage mit An- und Ausziehhilfen erwünscht und möglich ist. Es konnte in Studien gezeigt werden, dass sich durch den Einsatz verschiedener An- und Ausziehhilfen der Anteil der Patienten, die medizinische Kompressionsstrümpfe anlegen können, um etwa $20 \%$ steigerte [19]. Zudem schont der Einsatz von An- und Ausziehhilfen die Materialien, verbessert deren Haltbarkeit und erhöht die Adhärenz der Patienten [20].

Der große Vorteil der adaptiven Kompressionsbandagen wird insbesondere bei Patienten deutlich, die eine Selbstanlage, ggf. mit Unterstützung der Angehörigen, durchführen können. Hierdurch werden enorme Kosten für die Pflege eingespart. Da die Systeme sowohl für die Entstauungs- als auch Erhaltungsphase genutzt werden können, wird ein Wechsel der Materialien auch in einem Behandlungszeitraum von bis zu sechs Monaten nicht unbedingt notwendig. Diese Systeme können zudem im Laufe des Tages nachjustiert werden, wenn es bereits zu einer effektiven Ödemreduktion gekommen ist. Hierdurch kann die Effektivität der Kompressionstherapie verbessert werden [13, 21].

\section{Limitationen}

Bei der Berechnung von standardisierten Szenarien können nicht immer alle individuellen Faktoren erfasst werden. Wir haben daher in der Autorengruppe auf der Basis von Erfahrungswerten versucht, Standardszenarien zu formulieren, die einen Großteil der Patienten mit Ulcus cruris und Kompressionstherapie in Deutschland abbilden. Es gibt natürlich auch Patienten, die adaptive Kompressionsbandagen nicht selbstständig anlegen können, oder Patienten, die mithilfe ihrer Angehörigen ohne Pflegedienst phlebologische Kompressionsverbände adäquat applizieren.

Die Kosten für Materialien und das Personal sind, abhängig von den Einkaufspreisen und den jeweiligen Gebührenordnungen, beziehungsweise den zugrunde gelegten Gehaltsstrukturen, unterschiedlich. Wir haben für unsere Berechnungen Kosten von exemplarisch ausgewählten Produkten und keine durchschnittlichen Preise von Produktgruppen ausgewählt. Die Kosten können bei verschiedenen Herstellern durchaus variieren. Insbesondere bei den An- und Ausziehhilfen gibt es mehrere unterschiedliche Produktvarianten, sodass hier die Preisunterschiede am größten sind. Wir haben daher ein Produkt ausgewählt, das preislich im Mittelfeld der Kosten lag.

Bei den meisten Patienten mit Ulcus cruris muss zeitgleich eine Wundtherapie durchgeführt werden [22]. Hierfür kann es häufiger sinnvoll sein, ambulant einen Pflegdienst zu involvieren. Zudem kann durch eine adäquate Kompressionstherapie der Behandlungszeitraum für die Wundbehandlung erheblich beeinflusst werden. Die hier entstehenden Kosten haben wir nicht berücksichtigt.

In den Berechnungen wurden verschiedene indirekte Kosten für Ärzte, Krankenkasse oder Ausfallzeiten für Arbeitgeber ebenso wie Kosten für Patienten durch Reinigung wiederverwendbarer Materialien oder Zuzahlungen nicht berücksichtigt.

Ein optimal diagnostiziertes und therapiertes Ulcus cruris kann innerhalb von sechs Monaten bereits vollständig abgeheilt sein. Insbesondere Patienten mit CVI sollten anschließend als Rezidivprophylaxe medizinische Kompressionsstrümpfe (MKS) erhalten [23].

\section{KERNAUSSAGEN}

- Es gibt keine Methode der Kompressionsversorgung, die für alle Patienten mit Ulcus cruris und Ödemen medizinisch oder wirtschaftlich sinnvoll ist.

- Kompressionsverbände mit Kurzzugbinden sind sehr fehleranfällig und zeitaufwendig.

- „Neue“ und oft sinnvolle Alternativen sind Mehrkomponentensysteme, adaptive Kompressionsbandagen und Ulkus-Strumpfsysteme.

- Wann immer möglich sollte das Selbstmanagement der Patienten unterstützt werden. Hierfür sind Edukation und Hilfsmittel erforderlich.

- Bei der Verordnung sollten sowohl die Bedürfnisse und Fähigkeiten der Patienten als auch ökonomische Aspekte bekannt sein und berücksichtigt werden. 


\section{Interessenkonflikt}

Joachim Dissemond hat Honorare für Vorträge, Beratungen oder Unterstützung für Forschung von folgenden Firmen, die Materialien für Kompressionstherapie herstellen, erhalten: BSN medical, Paul Hartmann AG, medi, Juzo, Lohmann\&Rauscher, medi, Smith\&Nephew, Urgo. Kerstin Protz hat Honorare für Beratungen und/oder Vorträge von folgenden Firmen, die Materialien für Kompressionstherapie herstellen, erhalten: 3 M Medica, BSN medical, Paul Hartmann AG, Lohmann\& Rauscher, URGO, Smith\&Nephew, medi, Bauerfeind, Bösl Medizintechnik. Maurice Moelleken: keine.

Knut Kröger hat Honorare für Vorträge, Beratungen oder Unterstützung für Forschung von folgenden Firmen, die Materialien für Kompressionstherapie herstellen, erhalten: Sanofi, UCB, Bayer, Pfizer, daiichi sankyo, medi, Hartmann, Lohmann\&Rauscher, Urgo.

Literatur

[1] Dissemond J, Bültemann A, Gerber V et al. Definitionen für die Wundbehandlung. Hautarzt 2016; 67: 265-266

[2] Körber A, Klode J, Al-Benna S et al. Genese des chronischen Ulcus cruris bei 31619 Patienten im Rahmen einer Expertenbefragung in Deutschland. J Dtsch Dermatol Ges 2011; 9: 116-122

[3] Dissemond J, Storck M, Kröger K et al. Indikationen und Kontraindikationen der Kompressionstherapie bei chronischem Ulcus cruris. Wien Med Wochenschr (im Druck)

[4] Reich-Schupke S, Protz K, Dissemond I et al. Neue Entwicklungen in der phlebologischen Kompressionstherapie. Dtsch Med Wochenschr 2017; 142: 679-686

[5] Dissemond ], Assenheimer B, Bültemann A et al. Kompressionstherapie bei Patienten mit Ulcus cruris venosum. J Dtsch Dermatol Ges 2016; 14: 1072-1087

[6] Heyer K, Protz K, Augustin M. Compression therapy - cross-sectional observational survey about knowledge and practical treatment of specialised and non-specialised nurses and therapists. Int Wound J. (im Druck)

[7] Protz K, Heyer K, Dissemond J et al. Kompressionstherapie - Versorgungspraxis in Deutschland. Informationsstand von Patienten mit Ulcus cruris venosum über ihre Versorgung. J Dtsch Dermatol Ges 2016; 14: $1273-1282$

[8] O’Meara S, Cullum N, Nelson EA et al. Compression for venous leg ulcers. Cochrane Database Syst Rev 2012; 11: CD000265

[9] Weller CD, Buchbinder R, Johnston RV. Interventions for helping people adhere to compression treatments for venous leg ulceration. Cochrane Database Syst Rev 2016; 3: CD008378
[10] Renner R, Gebhardt C, Simon JC. Compliance hinsichtlich Kompressionstherapie bei Patienten mit floridem Ulcus cruris venosum. Med Klin 2010; 105: 1-6

[11] Stoffels-Weindorf M, Stoffels I, Jockenhöfer F et al. Qualität von Kompressionsverbänden, die von Patienten mit chronischem Ulcus cruris venosum selber angelegt wurden: Resultate einer prospektiven klinischen Studie. Hautarzt (im Druck)

[12] Weindorf M, Stoffels I, Schadendorf D et al. Einfluss visueller Kontrollsysteme auf die Effektivität von Kompressionsbehandlungen: Erste Resultate einer prospektiven klinischen Untersuchung verschiedener Probandenkollektive. Phlebologie 2012; 41: 18-24

[13] Mosti G, Partsch H. Druckmessungen unter Klettverschluss-Kompression - Selbstbehandlung durch feste, unelastische Beinwickelung. Vasomed 2017: 29: 212-216

[14] Ashby RL, Gabe R, Ali S et al. Clinical and cost-effectiveness of compression hosiery versus compression bandages in the treatment of venous leg ulcers (Venous leg Ulcer Study IV, VenUS IV): a randomised controlled trial. Lancet 2014; 383: 871-879

[15] Protz K, Reich-Schupke S, Klode K et al. Kompressionsmittel für die Entstauungstherapie - Eine vergleichende Erhebung im Querschnitt zu Handhabung, Anpressdruck und Tragegefühl. Hautarzt (im Druck)

[16] Dissemond J, Protz K, Hug J et al. Patientengerechte Versorgung - Ein Konzept der individualisierten Kompressionstherapie. Vasomed 2017; 29: 252-253

[17] Protz K, Dissemond J, Lulay G et al. Praxisprobleme und Therapiehürden Kompressionstherapie bei Ulcus cruris venosum. Pflege Z 2016; 69: 335340

[18] Partsch H, Mortimer P. Compression for leg wounds. Br J Dermatol 2015; 173: 359-369

[19] Sippel K, Seifert B, Hafner J. Donning devices (foot slips and frames) enable elderly people with severe chronic venous insufficiency to put on compression stockings. Eur J Vasc Endovasc Surg 2015; 49: 221-229

[20] Dissemond ], Protz K, Reich-Schupke S et al. Kompressionstherapie bei Ulcus cruris. Hautarzt 2016; 67: 311-325

[21] Mosti G, Cavezzi A, Partsch H et al. Adjustable velcro compression devices are more effective than inelastic bandages in reducing venous edema in the initial treatment phase: A randomized controlled trial. Eur J Vasc Endovasc Surg 2015; 50: 368-374

[22] Dissemond J, Augustin M, Eming S et al. Moderne Wundtherapie praktische Aspekte der lokalen, nicht-interventionellen Behandlung chronischer Wunden. J Dtsch Dermatol Ges 2014; 12: 541-554

[23] Nelson EA, Bell-Syer SE. Compression for preventing recurrence of venous ulcers. Cochrane Database Syst Rev 2014; 9: CD002303 